\title{
The influence of childhood abuse, adult life events, and affective temperaments on the well-being of the general, nonclinical adult population
}

This article was published in the following Dove Press journal:

Neuropsychiatric Disease and Treatment

12 April 2016

Number of times this article has been viewed

\author{
Yoshiaki Kanai ${ }^{1,2}$ \\ Yoshikazu Takaesu' \\ Yukiei Nakai ${ }^{3}$ \\ Masahiko Ichiki' \\ Mitsuhiko Sato' \\ Yasunori Matsumoto' \\ Jun Ishikawa' \\ Yasuyuki Ono' \\ Akiko Murakoshi \\ Hajime Tanabe ${ }^{4}$ \\ Ichiro Kusumi ${ }^{3}$ \\ Takeshi Inoue' \\ 'Department of Psychiatry, Tokyo \\ Medical University, ${ }^{2}$ Department of \\ Palliative Medicine, The University of \\ Tokyo Hospital, Tokyo, ${ }^{3}$ Department \\ of Psychiatry, Hokkaido University \\ Graduate School of Medicine, \\ Sapporo, ${ }^{4}$ Department of Clinical \\ Human Sciences, Graduate School \\ of Humanities and Social Sciences, \\ Shizuoka University, Shizuoka, Japan
}

Correspondence: Takeshi Inoue Department of Psychiatry, Tokyo Medical University, 6-7-I, Nishishinjuku, Shinjuku-ku, Tokyo 160-0023, Japan Tel +8I 33342 6I II

Fax +8I 333404499

Email tinoue@tokyo-med.ac.jp
Background: Previous studies have shown the effects of childhood abuse, life events, and temperaments on well-being (positive affect) and ill-being (negative affect). We hypothesized that childhood abuse, affective temperaments, and adult life events interact with one another and influence positive and negative affects in the general adult population and tested this hypothesis using structural equation modeling.

Methods: A total of 415 participants from the general, nonclinical adult population were studied using the following self-administered questionnaires: the Subjective Well-Being Inventory (SUBI); Life Experiences Survey (LES); Temperament Evaluation of the Memphis, Pisa, Paris, and San Diego Auto-questionnaire (TEMPS-A); and the Child Abuse and Trauma Scale (CATS). The data were analyzed with single and multiple regression analyses and structural equation modeling (Mplus).

Results: Childhood abuse indirectly predicted the worsening of positive and negative affects through cyclothymic, anxious, and irritable temperaments as measured by the TEMPS-A in the structural equation model. The cyclothymic, anxious, and irritable temperaments directly worsened the positive and negative affects and the negative appraisal of life events that occurred during the past year, while the hyperthymic temperament had the opposite effects.

Limitations: The subjects of this study were nonclinical volunteers. The findings might not be generalizable to psychiatric patients.

Conclusion: This study demonstrated that childhood abuse, particularly neglect, indirectly worsened the well-being of individuals through cyclothymic, anxious, and irritable affective temperaments. An important "mediator" role of affective temperaments in the effect of childhood abuse on well-being was suggested.

Keywords: childhood abuse, well-being, affective temperament, TEMPS-A, adult life events, structural equation model

\section{Introduction}

The constitution of the World Health Organization defines that health is a state of complete physical, mental, and social well-being and not merely the absence of disease or infirmity. ${ }^{1}$ In mental health and psychiatry, ill-being (negative or unpleasant affect), such as depression, anxiety, and tiredness, has been studied thoroughly, but the literature on well-being (positive or pleasant affect) is scarce. Positive and negative affects are reportedly only slightly negatively correlated or uncorrelated. ${ }^{2,3}$ This finding reminds us that the presence of positive affect (ie, happiness) is not the same as the absence of negative affect (ie, not depressed or upset). Accordingly, the effects of various factors that may influence mental health should be investigated on not only negative affect but also positive affect. 
Using structural equation modeling (SEM), we recently found that four of the five affective temperaments measured by the Temperament Evaluation of Memphis, Pisa, Paris, and San Diego Auto-questionnaire version (TEMPS-A) ${ }^{4}$ are strong "mediators" between childhood abuse and depressive symptoms in the general, nonclinical adult population. ${ }^{5}$ Childhood abuse indirectly predicts the severity of the depressive symptoms through four affective temperaments - depressive, cyclothymic, irritable, and anxious, while the hyperthymic temperament is not a mediator. These four temperaments depressive, cyclothymic, irritable, and anxious - directly predict the severity of the depressive symptoms and the negative appraisal of the life events that occurred during the past year. The negative appraisal of life events that occurred during the past year mildly, but significantly, predicts the severity of the depressive symptoms. Although the character profiles of the Temperament and Character Inventory are differentially associated with positive and negative affects, ${ }^{3}$ the combined effect of the affective temperaments of the TEMPS-A, childhood abuse, and life events on positive affect has not been reported.

We hypothesized that childhood abuse, adult life events (events within the past year), and affective temperaments interact with one another and influence well-being (positive affect) and ill-being (negative affect). The temperaments identified on the TEMPS-A are the putative "fundamental states" that Kraepelin considered to be enduring subclinical states in the absence of or before the florid symptoms of manic-depressive illness. ${ }^{6}$ For this reason, the factor "temperament" or TEMPS-A was located between two factors, childhood abuse and adult life events or positive/ negative affect. In this study, the interaction and effect of these three factors on well-being and ill-being in the general adult population were examined using the Subjective Well-Being Inventory (SUBI) as an autoquestionnaire of well-being (positive affect) and ill-being (negative affect). ${ }^{7}$ The covariance structure analysis was used to analyze this sophisticated interaction model.

\section{Subjects and methods Subjects}

This research was conducted during January 2014 and August 2014 on 853 Japanese volunteers from the general adult population. All volunteers were recruited by flyers and word of mouth. Of the 853 volunteers, 415 subjects (48.7\%) provided a complete response to the questionnaires. Five questionnaires, which are described in the following section, and a questionnaire on demographic data (sex, age, education, marital status, family members, employment status, past history of physical and psychiatric diseases, family history of psychiatric diseases, and subjective stratum identification [lowest social class $=10$ to highest social class $=1$ ]) were distributed. The completed questionnaires were returned anonymously to the research group by mail for complete confidentiality. Written informed consent was obtained from all of the subjects. This study was performed in accordance with the Declaration of Helsinki and was approved by the institutional review board of Hokkaido University Hospital and Tokyo Medical University.

\section{Questionnaires}

\section{LES}

The Life Experiences Survey (LES) is a 57-item self-report measure that allows respondents to indicate events that they have experienced during the past year. ${ }^{8}$ The format of the LES calls for subjects to separately rate the desirability and effect of the events that they have experienced. They are asked to indicate the events they experienced during the past year (0-6 months or 7 months-1 year) as well as 1 ) whether they viewed the event as being positive or negative and 2) the perceived impact of the particular event on their life at the time it occurred. The ratings are on a seven-point scale, ranging from extremely negative ( -3$)$ to extremely positive (+3). The sum of the impact ratings of the events that the subject designated as positive provides a "positive change score". A "negative change score" is derived by adding the impact ratings of the events that the subject designated as negative. This study used the Japanese version of the LES. ${ }^{5}$

\section{TEMPS-A}

The TEMPS-A is a self-rating questionnaire consisting of 109 items for men and 110 items for women. ${ }^{4}$ The subjects completed the Japanese standardized version of the TEMPS-A, which is a true $(=2) /$ false $(=1)$ questionnaire measuring the following temperament dimensions: depressive, cyclothymic, hyperthymic, irritable, and anxious. ${ }^{9}$ The score for each temperament subscale is the mean score on the items that comprise that subscale.

\section{CATS}

The Child Abuse and Trauma Scale (CATS) is a 38-item scale. Initial findings have demonstrated that this measure has strong internal consistency (Cronbach's $\alpha=0.63-0.90$ ) and test-retest reliability $(r=0.71-0.91) .{ }^{10}$ The CATS has been shown to significantly correlate with outcome measures such as dissociation, depression, stressful life events, and interpersonal difficulties. On each item, the participants rate how 
frequently they experienced a particular abusive experience during their childhood and adolescence using a scale of $0-4(0=$ never; $4=$ always $)$. The score for each subscale is the mean score of the items that comprise that subscale. There are three subscales that measure subjective reports of three aspects of adverse childhood experiences: neglect/negative home atmosphere, punishment, and sexual abuse.

Hajime Tanabe, one of the authors, developed and validated the Japanese version of the CATS by the classic translation-back-translation technique, with the permission and confirmation of Dr Sanders, the developer of the CATS. ${ }^{11}$

\section{SUBI}

The SUBI is a 40-item self-report measure designed to assess subjective well-being and ill-being. ${ }^{7}$ The score for each question ranges from 1 to 3 . The SUBI measures subjective well-being, ie, "positive affect" (19 items; happiness, life satisfaction, etc) and subjective ill-being, ie, "negative affect" (21 items; worry, anxiety, deficiency in social contacts, etc). Both subscales indicate that high scores represent a good state. The reliability and validity of the Japanese version were confirmed by Tonan et al. ${ }^{12}$

\section{PHQ-9}

The Japanese version of the Patient Health Questionnaire-9 (PHQ-9) was self-administered by the respondent in the written form. ${ }^{13}$ Major depressive episodes can be diagnosed in two ways using the PHQ-9: diagnostic algorithm and a summary score. This study used a summary score for assessing the severity of depressive symptoms.

\section{Data analysis}

We designed a structural equation model in which the positive and negative affects were predicted by childhood abuse, affective temperaments, and adult life events. Two latent variables, CATS (childhood abuse) and CAI-TEMPS-A (cyclothymic, anxious, and irritable temperaments), were composed of three observed variables that were evinced from the original questionnaire subscales. We used Mplus version 7.3 (Muthén \& Muthén, Los Angeles, CA, USA) to perform this path analysis to obtain the direct and indirect effects of all of the variables, and we used the robust maximum likelihood estimation to analyze the model. For the inferential statistical evaluation of SEM, we calculated the indices of goodness of fit using the Comparative Fit Index (CFI), the Tucker-Lewis Index (TLI), and the Root Mean Square Error of Approximation (RMSEA). According to the conventional criteria, a CFI $>0.95$, a TLI $>0.95$, and an RMSEA $<0.08$ indicate an acceptable fit; a CFI $>0.97$, a TLI $>0.97$, and an RMSEA $<0.05$ indicate a good fit. ${ }^{14}$ We standardized and indicated all of the coefficients (with a maximum of +1 and a minimum of -1 ) for the covariance structure analysis.

We conducted the Mann-Whitney $U$-test to compare demographic characteristics and the questionnaire data between the two groups. Spearman's rank correlation coefficient and multiple regression analysis were used for correlations between the parameters and the predictive factors.

The statistical analyses were conducted using Mplus version 7.3 for the covariance structure analysis, Excel Statistics for Macintosh (Esumi Co, Ltd, Tokyo, Japan) for the multiple regression analysis, and 4-Steps Excel Statistics (The publisher OMS Ltd., Saitama, Japan) for Spearman's rank correlation coefficient and the Mann-Whitney $U$-test.

The differences were considered to be statistically significant at $P<0.05$.

\section{Results}

\section{The subjects' demographic characteristics and PHQ-9, SUBI, CATS, TEMPS-A, and LES scores}

The demographic characteristics and PHQ-9, SUBI, CATS, TEMPS-A, and LES scores of 415 subjects are presented in Tables 1 and 2. Age; education (years); subjective stratum identification; the PHQ-9 summary scores; the negative affect scores of the SUBI; neglect, punishment, and total scores of the CATS; the depressive, cyclothymic, anxious, hyperthymic, and irritable temperament scores of the TEMPS-A; and the negative and positive change scores of the LES were significantly correlated with the positive affect scores of the SUBI, as determined by the Spearman's rank correlation coefficients (Table 1).

Sex (male), marital status (married), and the absence of a first-degree relative with psychiatric disease were associated with high SUBI negative affect scores, as determined by the Mann-Whitney $U$-test, suggesting that these factors are associated with low ill-being or negative affect because high negative affect scores indicate low negative affect in the SUBI (Table 2). Age; education (years); number of offspring; subjective stratum identification; the PHQ-9 summary scores; the positive affect scores of the SUBI; sexual abuse, neglect, punishment, and total scores of the CATS; the depressive, cyclothymic, hyperthymic, anxious, and irritable temperament scores of the TEMPS-A; and the 
Table I Characteristics, SUBI, CATS, TEMPS-A, and LES and correlation with the SUBI positive affect scores or effects on the SUBI positive affect scores in 415 general adult subjects

\begin{tabular}{|c|c|c|}
\hline Characteristics or measures & $\begin{array}{l}\text { Value (number or } \\
\text { mean } \pm S D \text { ) }\end{array}$ & $\begin{array}{l}\text { Correlation with SUBI positive affect scores }(\rho) \text { or effect } \\
\text { on them (mean } \pm \text { SD of positive affect scores, } U \text {-test) }\end{array}$ \\
\hline Age & $42.3 \pm 12.0$ & $\rho=-0.17 * *$ \\
\hline Sex (male:female) & $222: 193$ & Male $39.4 \pm 6.5$ vs female $38.6 \pm 6.5$, ns (U-test) \\
\hline Education, years & $\mid 5.1 \pm 2.0$ & $\rho=0.17^{* *}$ \\
\hline Employment status (employed:unemployed) & $352: 56$ & Employed $38.9 \pm 6.4$ vs unemployed $39.6 \pm 7.4$, ns (U-test) \\
\hline Marital status (married:unmarried) & $291: 121$ & Married $39.3 \pm 6.4$ vs unmarried $38.3 \pm 6.7$, ns (U-test) \\
\hline Number of cohabiters & $1.8 \pm 1.5$ & $\rho=0.04, \mathrm{~ns}$ \\
\hline Number of offspring & $1.3 \pm 1.2$ & $\rho=0.04, \mathrm{~ns}$ \\
\hline Comorbidity of physical disease (yes:no) & $83: 328$ & Yes $38.1 \pm 6.4$ vs no $39.3 \pm 6.5$, ns (U-test) \\
\hline First-degree relative with psychiatric diseases (yes:no) & $41: 372$ & Yes $37.9 \pm 7.1$ vs no $39.1 \pm 6.5$, ns (U-test) \\
\hline Subjective stratum identification & $4.9 \pm 1.5$ & $\rho=-0.19 * *$ \\
\hline PHQ-9 summary score & $3.3 \pm 4.0$ & $\rho=-0.35 * *$ \\
\hline SUBI positive affect score & $39.0 \pm 6.5$ & \\
\hline SUBI negative affect score & $52.1 \pm 6.4$ & $\rho=-0.47^{* *}$ \\
\hline \multicolumn{3}{|l|}{ CATS (average score) } \\
\hline Sexual abuse & $0.04 \pm 0.22$ & $\rho=-0.03, \mathrm{~ns}$ \\
\hline Neglect & $0.62 \pm 0.58$ & $\rho=-0.2 I^{* *}$ \\
\hline Punishment & $1.42 \pm 0.62$ & $\rho=-0.14^{* *}$ \\
\hline Total & $0.72 \pm 1.32$ & $\rho=-0.20 * *$ \\
\hline \multicolumn{3}{|l|}{ TEMPS-A (average score) } \\
\hline Depressive & $1.37 \pm 0.16$ & $\rho=-0.40 * *$ \\
\hline Cyclothymic & $1.20 \pm 0.18$ & $\rho=-0.25^{* *}$ \\
\hline Hyperthymic & $1.27 \pm 0.18$ & $\rho=0.45^{* *}$ \\
\hline Anxious & $1.19 \pm 0.19$ & $\rho=-0.30 * *$ \\
\hline Irritable & $1.14 \pm 0.15$ & $\rho=-0.28 * *$ \\
\hline \multicolumn{3}{|l|}{ LES (change score) } \\
\hline Negative & $1.64 \pm 3.09$ & $\rho=-0.14^{* *}$ \\
\hline Positive & $1.63 \pm 2.92$ & $\rho=0.19 * *$ \\
\hline
\end{tabular}

Notes: Data are presented as mean \pm SD or numbers; $\rho=$ Spearman's rank correlation coefficient; $* * P<0.0$; high positive affect scores and high negative affect scores indicate high positive affect and low negative affect, respectively.

Abbreviations: PHQ-9, Patient Health Questionnaire-9; CATS, Child Abuse and Trauma Scale; TEMPS-A, Temperament Evaluation of the Memphis, Pisa, Paris, and San Diego Auto-questionnaire; LES, Life Experiences Survey; SUBI, Subjective Well-Being Inventory; SD, standard deviation; ns, not significant.

Table 2 Characteristics, SUBI, CATS, TEMPS-A, and LES and correlation with the SUBI negative affect scores or effects on the SUBI negative affect scores in 415 general adult subjects

\begin{tabular}{|c|c|c|}
\hline Characteristics or measures & $\begin{array}{l}\text { Value (number } \\
\text { or mean } \pm \text { SD) }\end{array}$ & $\begin{array}{l}\text { Correlation with SUBI negative affect scores }(\rho) \text { or effect } \\
\text { on them (mean } \pm \text { SD of negative affect scores, } U \text {-test) }\end{array}$ \\
\hline Age & $42.3 \pm 12.0$ & $\rho=0.17 * *$ \\
\hline Sex (male:female) & $222: 193$ & Male $53.3 \pm 5.7$ vs female $50.8 \pm 6.9 * *$ (U-test) \\
\hline Education, years & $\mid 5.1 \pm 2.0$ & $\rho=0.12^{*}$ \\
\hline Employment status (employed:unemployed) & $352: 56$ & Employed $52.3 \pm 6.3$ vs unemployed $50.8 \pm 7.0$, ns (U-test) \\
\hline Marital status (married:unmarried) & 291:121 & Married $53.0 \pm 5.9$ vs unmarried $49.8 \pm 7.0 * *$ (U-test) \\
\hline Number of cohabiters & $1.8 \pm 1.5$ & $\rho=0.02, \mathrm{~ns}$ \\
\hline Number of offspring & $1.3 \pm 1.2$ & $\rho=0.18^{* *}$ \\
\hline Comorbidity of physical disease (yes:no) & $83: 328$ & Yes $52.0 \pm 6.6$ vs no $52.2 \pm 6.3$, ns (U-test) \\
\hline $\begin{array}{l}\text { First-degree relative with psychiatric } \\
\text { diseases (yes:no) }\end{array}$ & $41: 372$ & Yes $50.2 \pm 7.0$ vs no $52.3 \pm 6.3^{*}$ (U-test) \\
\hline Subjective stratum identification & $4.9 \pm 1.5$ & $\rho=-0.17^{* *}$ \\
\hline PHQ-9 summary score & $3.3 \pm 4.0$ & $\rho=-0.6 \mathrm{I}^{* *}$ \\
\hline SUBI positive affect score & $39.0 \pm 6.5$ & $\rho=-0.47 * *$ \\
\hline SUBI negative affect score & $52.1 \pm 6.4$ & \\
\hline \multicolumn{3}{|l|}{ CATS (average score) } \\
\hline Sexual abuse & $0.04 \pm 0.22$ & $\rho=-0.16 * *$ \\
\hline
\end{tabular}


Table 2 (Continued)

\begin{tabular}{lll}
\hline Characteristics or measures & $\begin{array}{l}\text { Value (number } \\
\text { or mean } \pm \text { SD) }\end{array}$ & $\begin{array}{l}\text { Correlation with SUBI negative affect scores }(\rho) \text { or effect } \\
\text { on them (mean } \pm \text { SD of negative affect scores, U-test) }\end{array}$ \\
\hline Neglect & $0.62 \pm 0.58$ & $\rho=-0.41^{* *}$ \\
Punishment & $1.42 \pm 0.62$ & $\rho=-0.23^{* *}$ \\
Total & $0.72 \pm 1.32$ & $\rho=-0.39^{* *}$ \\
TEMPS-A (average score) & $1.37 \pm 0.16$ & $\rho=-0.42^{* *}$ \\
Depressive & $1.20 \pm 0.18$ & $\rho=-0.55^{* *}$ \\
Cyclothymic & $1.27 \pm 0.18$ & $\rho=0.20^{* *}$ \\
Hyperthymic & $1.19 \pm 0.19$ & $\rho=-0.58^{* *}$ \\
Anxious & $1.14 \pm 0.15$ & $\rho=-0.44^{* *}$ \\
Irritable & & $\rho=0.26^{* *}$ \\
LES (change score) & $1.64 \pm 3.09$ & $\rho=0.04, \mathrm{~ns}$ \\
Negative & $1.63 \pm 2.92$ & \\
Positive & Sp & \\
\hline
\end{tabular}

Notes: Data are presented as mean \pm SD or numbers; $\rho=$ Spearman's rank correlation coefficient; $* P<0.05$, $* * p<0.0$ I; high positive affect scores and high negative affect scores indicate high positive affect and low negative affect, respectively.

Abbreviations: PHQ-9, Patient Health Questionnaire-9; CATS, Child Abuse and Trauma Scale; TEMPS-A, Temperament Evaluation of the Memphis, Pisa, Paris, and San Diego Auto-questionnaire; LES, Life Experiences Survey; SUBI, Subjective Well-Being Inventory; SD, standard deviation; ns, not significant.

negative, but not positive, change scores of the LES were significantly correlated with the negative affect scores of the SUBI, as determined by the Spearman's rank correlation coefficients (Table 2).

\section{Stepwise multiple regression analysis of the putative explanatory variables on the positive and negative affect scores of the SUBI}

The putative explanatory variables in Tables 1 and 2 that showed significant correlations with the positive and negative affect scores of the SUBI, as determined by the Spearman's rank correlation coefficients, or that had significant effects on positive and negative affect scores of the SUBI, as determined by the Mann-Whitney $U$-test, were further analyzed by a stepwise multiple regression analysis. The PHQ-9 summary score was not included as an explanatory variable because this variable falls into the same category as positive and negative affects of the SUBI.

Table 3 shows the results of the stepwise multiple regression analysis, where a positive affect score of the SUBI was the dependent factor and the subjects' age; education (years); subjective stratum identification (lowest $=10$ to highest =1); neglect, punishment, and sexual abuse scores of the CATS; hyperthymic, depressive, cyclothymic, anxious, and irritable temperament scores of the TEMPS-A; and negative and positive change scores of the LES were independent factors. A total score on the CATS that had a significant correlation with the positive affect scores (Table 1) was excluded from the stepwise multiple regression analysis because it had a high correlation with the neglect score on the CATS ( $\rho=0.86)$. When entering these independent factors into the stepwise multiple regression analysis, the subjects' age; neglect and sexual abuse scores on the CATS; hyperthymic, irritable, and depressive scores on the TEMPS-A; and a positive change score on the LES were significant predictors of a positive affect score on the SUBI $(F=35.1, P<0.0001$, adjusted $R^{2}=0.43$ ), whereas the other factors were excluded from the model. Multicollinearity was denied in this multiple regression analysis.

Table 4 shows the results of a stepwise multiple regression analysis, where a negative affect score of the SUBI was the dependent factor and the subjects' age; sex (male 0, female 1); education (years); marital status (unmarried 0 ,

Table 3 The results of a stepwise multiple regression analysis of the SUBI positive affect scores

\begin{tabular}{lll}
\hline Independent factors & Beta & P-value \\
\hline Hyperthymic score of TEMPS-A & 0.39 & $<0.000$ I \\
Irritable score of TEMPS-A & -0.24 & $<0.000$ I \\
Depressive score of TEMPS-A & -0.21 & $<0.000$ I \\
Neglect score of CATS & -0.17 & 0.0004 \\
Age & -0.12 & 0.0032 \\
Positive change score of LES & 0.11 & 0.0038 \\
Sexual abuse score of CATS & 0.09 & 0.0271 \\
Subjective stratum identification & -0.06 & 0.0904 \\
Punishment score of CATS & 0.07 & 0.0918 \\
Adjusted $R^{2}=0.43$ & & $<0.000$ I \\
\hline
\end{tabular}

Notes: Beta $=$ standardized partial regression coefficient; dependent factor: SUBI positive affect (well-being) score; thirteen independent factors: age; education (years); subjective stratum identification (lowest $=10$ to highest $=1$ ); neglect, punishment, and sexual abuse scores of CATS; hyperthymic, depressive, cyclothymic, anxious, and irritable temperament scores of TEMPS-A; and negative and positive change scores of LES.

Abbreviations: CATS, Child Abuse and Trauma Scale; TEMPS-A, Temperament Evaluation of the Memphis, Pisa, Paris, and San Diego Auto-questionnaire; LES, Life Experiences Survey; SUBI, Subjective Well-Being Inventory. 
Table 4 The results of a stepwise multiple regression analysis of the SUBI negative affect scores

\begin{tabular}{lll}
\hline Independent factors & Beta & $P$-value \\
\hline Anxious score of TEMPS-A & -0.32 & $<0.000$ I \\
Hyperthymic score of TEMPS-A & 0.20 & $<0.000$ I \\
Cyclothymic score of TEMPS-A & -0.23 & $<0.000$ I \\
Age & 0.11 & 0.0019 \\
Marital status (unmarried 0, married I) & 0.11 & 0.0030 \\
Positive change score of LES & 0.10 & 0.0070 \\
Irritable score of TEMPS-A & -0.12 & 0.0138 \\
Negative change score of LES & -0.09 & 0.0173 \\
Neglect score of CATS & -0.08 & 0.0505 \\
Sexual abuse score of CATS & -0.06 & 0.0953 \\
Adjusted $R^{2}=0.54$ & & $<0.000$ I \\
\hline
\end{tabular}

Notes: Beta $=$ standardized partial regression coefficient; dependent factor: SUBI negative affect (ill-being) score; seventeen independent factors: age; sex (male 0 , female I); education (years); marital status (unmarried 0 , married I); number of offspring; first-degree relative with psychiatric diseases (no 0 , yes I); subjective stratum identification (lowest $=10$ to highest $=1$ ); neglect, punishment, and sexual abuse scores of CATS; hyperthymic, depressive, cyclothymic, anxious, and irritable temperament scores of TEMPS-A; and negative and positive change scores of LES. Abbreviations: CATS, Child Abuse and Trauma Scale; TEMPS-A, Temperament Evaluation of the Memphis, Pisa, Paris, and San Diego Auto-questionnaire; LES, Life Experiences Survey; SUBI, Subjective Well-Being Inventory.

married 1); number of offspring; first-degree relative with psychiatric diseases (no 0 , yes 1); subjective stratum identification (lowest $=10$ to highest $=1$ ); neglect, punishment, and sexual abuse scores of the CATS; hyperthymic, depressive, cyclothymic, anxious, and irritable temperament scores of the TEMPS-A; and negative and positive change scores of the LES were independent factors. A total score on the CATS that had a significant correlation with the negative affect scores (Table 2) was excluded from the stepwise multiple regression analysis because it had a high correlation with the neglect score on the CATS $(\rho=0.86)$. When entering these independent factors in a stepwise multiple regression analysis, the subjects' age; marital status (married); anxious, hyperthymic, cyclothymic, and irritable scores on the TEMPS-A; and positive and negative change scores on the LES were significant predictors of a negative affect score on the SUBI $\left(F=48.5, P<0.0001\right.$, adjusted $\left.R^{2}=0.54\right)$, whereas the other factors were excluded from the model. Multicollinearity was denied in this multiple regression analysis.

\section{Analysis of the structural equation model}

To examine the complicated association between the CATS, TEMPS-A, LES, and SUBI scores, we built a structural equation model based on the results of the aforementioned univariate analyses and multiple regression analyses (Figure 1A and B). The results of the path coefficients calculated by Mplus are shown in Figure 1A and B.

A good fit of the model (Figure 1A) was obtained based on the following criteria: RMSEA $=0.050, \mathrm{CFI}=0.966$, and $\mathrm{TLI}=0.939$. The path coefficients with solid lines in Figure 1A

\section{A Positive affect (well-being)}

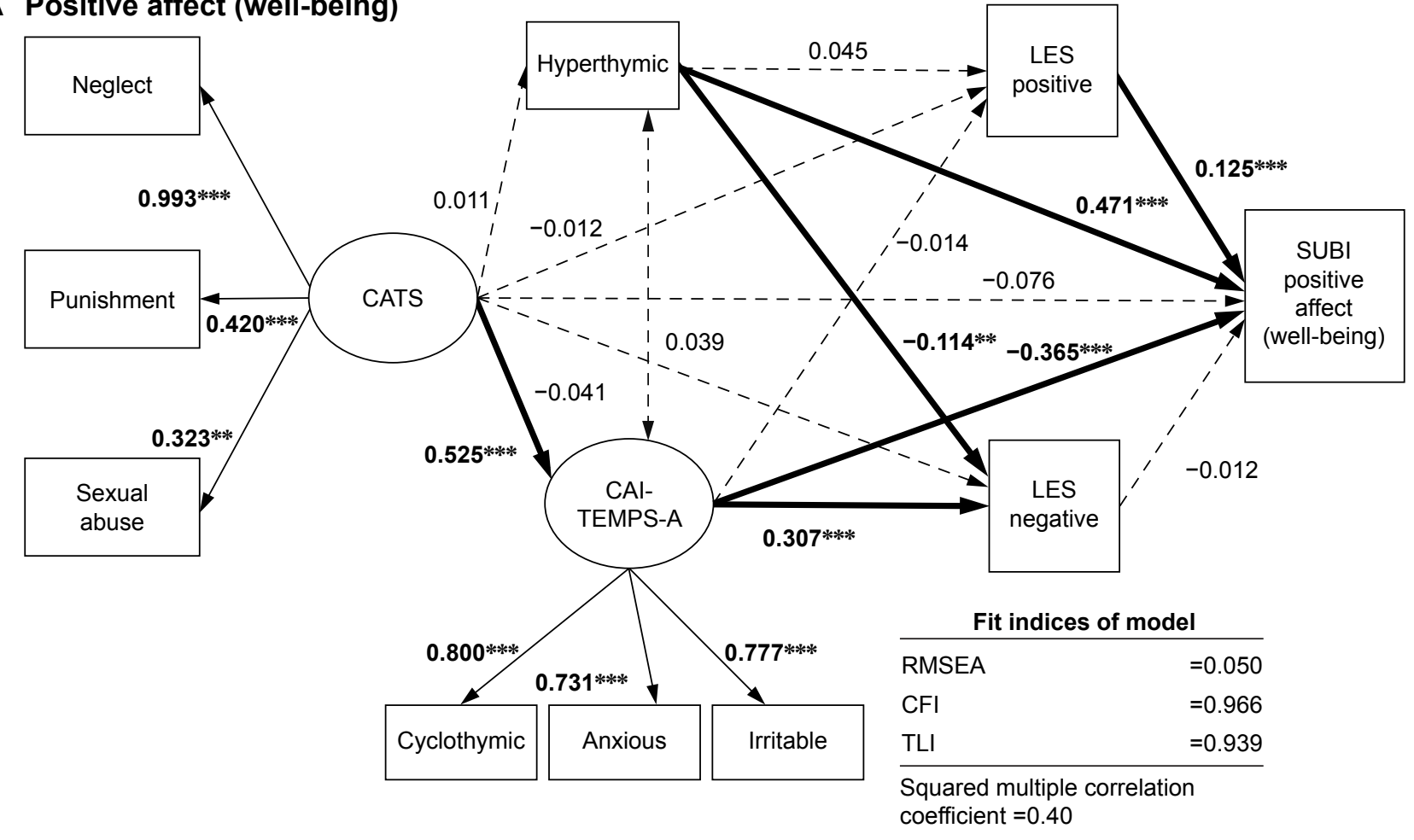

Figure I (Continued) 


\section{B Negative affect (ill-being)}

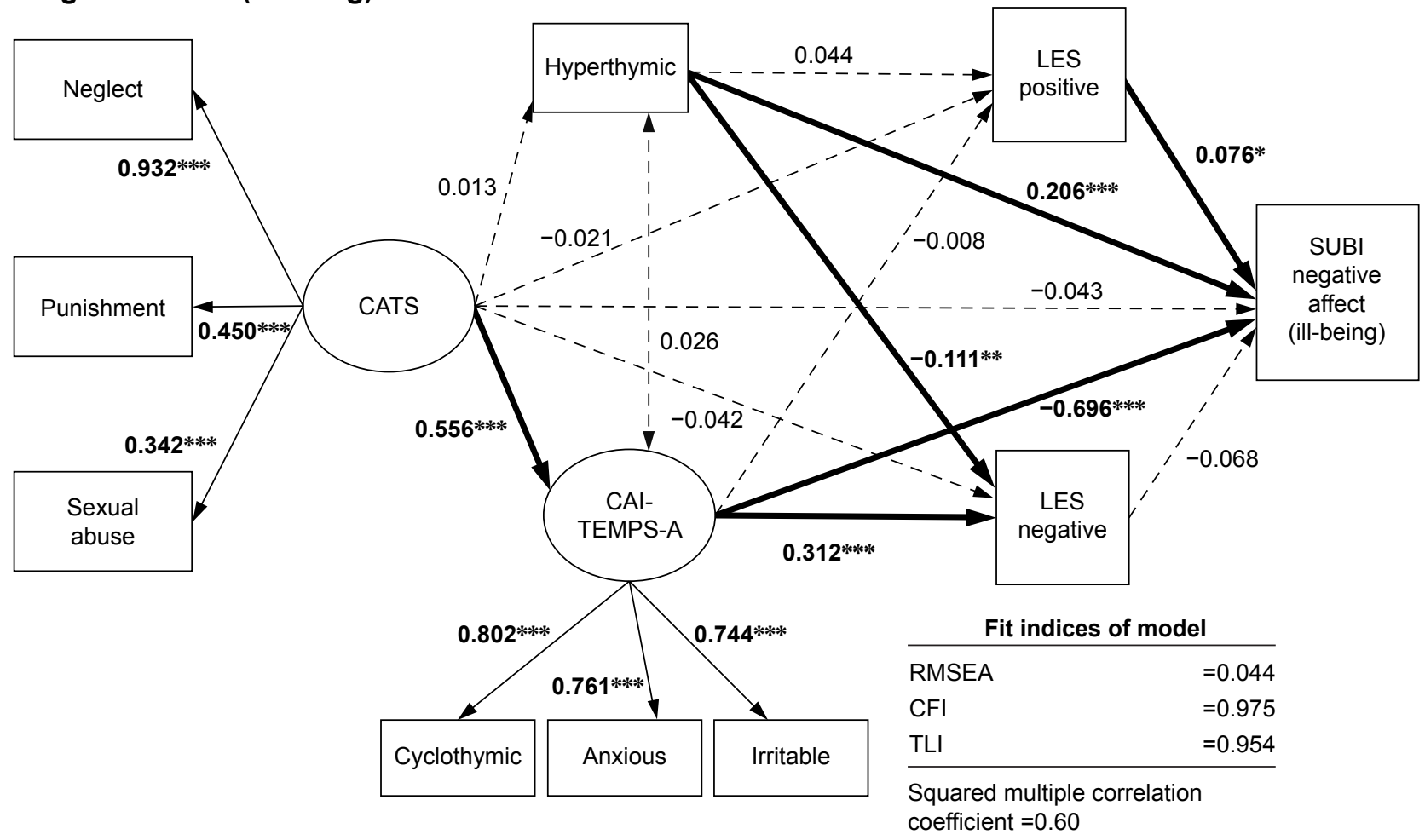

Figure I The results of the covariance structure analysis in the structural equation model, using the childhood abuse (CATS), affective temperaments (TEMPS-A), adult life events (LES), positive affect (well-being) (A) and negative affect (ill-being) (B) (SUBI) scores from 4I5 subjects from the general, nonclinical adult population.

Notes: The rectangles indicate the observed variables and the ovals indicate the latent variables. The arrows with solid lines represent the statistically significant paths, and the broken lines show the nonsignificant paths. The numbers beside the arrows show the standardized path coefficients (minimum $-\mathrm{I}$, maximum I). $* P<0.05$, $* * P<0.0 \mathrm{I}$, $* * * P<0.001$.

Abbreviations: CATS, Child Abuse and Trauma Scale; TEMPS-A, Temperament Evaluation of the Memphis, Pisa, Paris, and San Diego Auto-questionnaire; CAI, cyclothymic, anxious, and irritable temperaments; LES, Life Experiences Survey; SUBI, Subjective Well-Being Inventory; RMSEA, Root Mean Square Error of Approximation; CFI, Comparative Fit Index; TLI, Tucker-Lewis Index.

were significant $(P<0.05-0.001)$. According to the SEM and partially consistent with the results of the multiple regression analysis (Table 3), a positive affect score on the SUBI was significantly predicted by three temperament scores (cyclothymic, anxious, and irritable) and a hyperthymic temperament score on the TEMPS-A and a positive change score on the LES. The effect of the CATS subscales on a positive affect score on the SUBI was indirect and mediated by three temperament scores (cyclothymic, anxious, and irritable) on the TEMPS-A (indirect path coefficient $=-0.19$, $P<0.001)$. The effect of the CATS subscales on a negative change score on the LES was also indirect and mediated by three temperament scores (cyclothymic, anxious, and irritable) on the TEMPS-A (indirect path coefficient $=0.16$, $P<0.001)$. The hyperthymic temperament directly predicted a negative change score on the LES. However, a negative change score on the LES did not predict a positive affect score on the SUBI. The squared multiple correlation coefficient for a positive affect score on the SUBI was 0.40. In another model that added depressive temperament as the observed variable for the latent variable, including the cyclothymic, anxious, and irritable temperaments, the fit indices were not satisfactory (data not shown).

A good fit of the model (Figure 1B) was obtained using the following criteria: $\mathrm{RMSEA}=0.044, \mathrm{CFI}=0.975$, and TLI $=0.954$. The path coefficients with solid lines in Figure 1B were significant $(P<0.05-0.001)$. According to the SEM and partially consistent with the results of the multiple regression analysis (Table 4), a negative affect score on the SUBI was significantly predicted by three temperament scores (cyclothymic, anxious, and irritable) and a hyperthymic temperament score on the TEMPS-A and a positive change score on the LES. The effect of the CATS subscales on a negative affect score of the SUBI was indirect and mediated by three temperament scores (cyclothymic, anxious, and irritable) of the TEMPS-A (indirect path coefficient $=-0.39$, $P<0.001)$. The effect of the CATS subscales on a negative change score of the LES was indirect and mediated by three temperament scores (cyclothymic, anxious, and irritable) of the TEMPS-A (indirect path coefficient $=0.17, P<0.001$ ). 
The hyperthymic temperament directly predicted a negative change score on the LES. However, a negative change score on the LES did not predict a negative affect score on the SUBI. The squared multiple correlation coefficient for a negative affect score on the SUBI was 0.60. In another model that added depressive temperament as the observed variable for the latent variable, including the cyclothymic, anxious, and irritable temperaments, the fit indices were not satisfactory (data not shown).

\section{Discussion}

This study is the first report suggesting that childhood abuse indirectly predicted both positive affect (well-being) and negative affect (ill-being) in the general, nonclinical adult population through the affective temperaments measured by the TEMPS-A in the SEM. Three temperaments - cyclothymic, anxious, and irritable - directly predicted positive and negative affect and the negative change score on the LES during the past year; as these temperament scores increased, the positive and negative affect scores and the negative change score on the LES were worsened. Unexpectedly, the negative change score of the LES during the past year did not significantly influence either positive or negative affect, but the positive change score of the LES alleviated both positive and negative affects, independent of childhood abuse and affective temperaments. In contrast to the effects of the cyclothymic, irritable, and anxious temperaments, the hyperthymic temperament improved both positive and negative affects and the negative change score of the LES, independent of childhood abuse. The validity of this result was supported by the good fit indices of the model and partially by the results of the multiple regression analyses, which showed that childhood abuse, affective temperaments, and life events significantly predicted positive and negative affects, with the exception of the effect of childhood abuse on negative affect. Two models accounted for $40 \%$ of the variability in positive affect scores of the SUBI and $60 \%$ of the variability in negative affect scores of the SUBI. Taken together, the present study suggests that affective temperament has an important role as a mediator on the effect of childhood abuse on positive and negative affects. Most important, this study showed that the same SEM is applicable to positive and negative affects, although positive and negative affects are reportedly only slightly negatively correlated or uncorrelated. ${ }^{2}$

The very important significance of this SEM study was that the SEM analysis proved that childhood abuse is an indirect significant predictor of negative affect because the multiple regression analysis did not indicate that childhood abuse was a significant predictor of negative affect. Without the SEM analysis, our study might have led to the erroneous conclusion that childhood abuse is not associated with negative affect. The ability of the SEM analysis to identify indirect effects is its major advantage. Several studies reported that childhood abuse is associated with negative affect, including depressive and anxiety symptoms in nonclinical and clinical populations. ${ }^{5,10,15-19}$ Consistent with the results of this study, our previous study reported that childhood abuse indirectly increases depressive symptoms, negative affect, through the mediator role of affective temperament in the general, nonclinical adult population. ${ }^{5}$

In contrast to negative affect, the effect of stress in childhood and adulthood on positive affect, one of the various well-being indices, has been rarely investigated. A metaanalytic study showed that unemployment decreases life satisfaction and marital or family satisfaction, but did not mention the effect of unemployment on positive affect. ${ }^{20}$ A previous large-scale study with a multiple regression analysis demonstrated that absence of negative life events was positively associated with well-being. ${ }^{21}$ Accordingly, our present study first showed that childhood abuse and positive life events in adulthood indirectly decreased and directly increased positive affect, respectively. Unexpectedly, negative life events in adulthood did not affect positive affect in the SEM, inconsistent with the previous study. ${ }^{21}$ In our multiple regression analysis and SEM, other more potent factors, such as childhood abuse and temperaments, might have influenced relative contribution of negative life events to positive affect.

Similarly, there have been only a few studies that investigated the effect of positive adult life events on negative affect: positive life events decreased anxiety and depressive symptoms. ${ }^{22,23}$ Consistent with previous reports, this study also showed the decreasing effect of positive adult life events on negative affect, which is psychologically and clinically important. This effect encourages us to recommend that clients with depressive symptoms or negative affect experience positive adult life events. In this aspect, our recent study reported that positive adult life events had an inhibitory "moderator" effect on depressive symptoms that was increased by cyclothymic and anxious temperaments in the general, nonclinical adult population, although positive adult life events by themselves did not affect depressive symptoms. ${ }^{18}$

An association between character profiles and positive and negative affects was reported. ${ }^{3}$ Character profiles, as 
measured by the Temperament and Character Inventory, are differentially associated with positive and negative affects; among the three dimensions of character, self-directedness, cooperativeness, and self-transcendence, higher selfdirectedness and higher self-transcendence are associated with higher positive affect, while higher self-directedness is associated with lower negative affect. ${ }^{3}$ Because the five affective temperaments measured by the TEMPS-A are weakly or moderately correlated with self-directedness, cooperativeness, and self-transcendence, ${ }^{24}$ the affective temperaments on the TEMPS-A are predicted to be associated with positive and negative affects. This study is the first to show that the affective temperaments on the TEMPS-A are associated with positive and negative affects; the hyperthymic temperament improved both the positive and negative affects, but the cyclothymic, irritable, and anxious temperaments worsened both the positive and negative affects. It is interesting to note that the two groups of affective temperaments affect positive and negative affects differently. Consistent with our present and previous results, ${ }^{18}$ Rovai et a ${ }^{25}$ noted that there is a gap between the hyperthymic temperament, which must be considered the most functional and desirable temperament, and the cyclothymic, depressive, irritable, and anxious temperaments, which are more closely associated with mood, anxiety, and substance use disorders, thus implying that the individuals with these temperaments may have difficulty in adapting emotionally and behaviorally to somatic diseases and life stressors. There are sex differences in some affective temperaments: more hyperthymic temperament in males and more depressive and anxious temperaments in females (our unpublished data and previous studies). ${ }^{25}$ Two groups of affective temperaments may affect sex differences in depressive and anxiety symptoms and in the outcome of psychiatric disorders, such as depressive and anxiety disorders.

Among some demographic factors that showed significant effects on the positive and negative affect scores on the SUBI in single regression analyses, only age and marital status were significant in multiple regression analyses. A large-scale previous study with a multiple regression analysis reported that male, greater age, cohabiting, good childhood conditions, support from friends, sound financial situation, and absence of negative life events, but not education or country of origin, were positively correlated with well-being, ${ }^{21}$ partly consistent with our present results. Interestingly, in our study, age had opposite effects on positive and negative affects: age improved negative affect and worsened positive affect.

Because this study examined only the general, nonclinical adult population, which is a limitation of this study, the linkage between affective temperaments, childhood abuse, adult life events, and positive and negative affects should be studied further using a large sample size of psychiatric patients. Due to this limitation, the findings may not be generalizable to psychiatric patients. Another limitation of this study is that childhood abuse was estimated retrospectively. To confirm the influence of childhood abuse and temperaments on positive and negative affects, a prospective longitudinal study of a representative birth cohort is necessary.

\section{Conclusion}

In conclusion, using the structural equation model, this study suggested that childhood abuse, particularly neglect, heightens three temperaments (cyclothymic, anxious, and irritable), which, in turn, increase the negative appraisal of stressful life events and worsen positive and negative affects. On the other hand, the hyperthymic temperament and positive adult life events improve the positive and negative affects An important "mediator" role of the affective temperament in the effect of childhood abuse on positive and negative affects was suggested.

\section{Acknowledgments}

This study was partly supported by the program "Integrated research on neuropsychiatric disorders" conducted under the Strategic Research Program for Brain Sciences by the Ministry of Education, Culture, Sports, Science, and Technology of Japan.

\section{Disclosure}

The authors report no financial or other relationship that is relevant to the subject of this article. Dr Kanai reports a grant from the Japanese Society of Palliative Medicine. Dr Inoue reports personal fees from Otsuka Pharmaceutical, Pfizer, GlaxoSmithKline, Astellas, Eli Lilly, Mitsubishi Tanabe Pharma, Mochida Pharmaceutical, Meiji Seika Pharma, Asahi Kasei Pharma, Shionogi, Janssen Pharmaceutical, Takeda Pharmaceutical, MSD, Yoshitomiyakuhin, Dainippon Sumitomo Pharma, Eisai, and AbbVie GK; and grants from Otsuka Pharmaceutical, Eli Lilly, Eisai, Yoshitomiyakuhin, Mitsubishi Tanabe Pharma, AbbVie GK, Pfizer, Astellas, and Meiji Seika Pharma; and is a member of the advisory boards of GlaxoSmithKline, Pfizer, Eli Lilly, Mochida Pharmaceutical, and Mitsubishi Tanabe Pharma. Dr Takaesu reports personal fees from Otsuka Pharmaceutical, Meiji Seika Pharma, Eli Lilly, Eisai, Mitsubishi Tanabe Pharma, and Yoshitomiyakuhin; and grants from Otsuka Pharmaceutical, Meiji Seika Pharma, and Eisai. Dr Ichiki reports personal fees 
from Otsuka Pharmaceutical, Pfizer, Eli Lilly, Mitsubishi Tanabe Pharma, Mochida Pharmaceutical, Meiji Seika Pharma, Janssen Pharmaceutical, Takeda Pharmaceutical, MSD, Dainippon Sumitomo Pharma, and Eisai; and grants from Otsuka Pharmaceutical, Eli Lilly, Eisai, Shionogi, Takeda Pharmaceutical, MSD, and Pfizer; and is a member of the advisory board of Meiji Seika Pharma. Dr Kusumi reports personal fees from Astellas, Chugai Pharmaceutical, Dainippon Sumitomo Pharma, Eisai, Eli Lilly, Janssen Pharmaceutical, Kyowa Hakko Kirin, Meiji Seika Pharma, MSD, Nippon Chemiphar, Novartis Pharma, Ono Pharmaceutical, Otsuka Pharmaceutical, Pfizer, Tanabe Mitsubishi Pharma, Shionogi, and Yoshitomiyakuhin; grants from AbbVie GK, Asahi Kasei Pharma, and Boehringer Ingelheim; and grants and personal fees from Daiichi Sankyo, Dainippon Sumitomo Pharma, Eisai, Eli Lilly, GlaxoSmithKline, Kyowa Hakko Kirin, Meiji Seika Pharma, MSD, Novartis Pharma, Ono Pharmaceutical, Otsuka Pharmaceutical, Pfizer, Takeda Pharmaceutical, Mitsubishi Tanabe Pharma, Shionogi, and Yoshitomiyakuhin. The other authors report no conflicts of interest in this work.

\section{References}

1. World Health Organization. Definition of Health in Preamble to the Constitution of the World Health Organization. Geneva, Switzerland: World Health Organization; 1946.

2. Bradburn NM. The Structure of Psychological Well-Being. Chicago, IL: Aldine; 1969.

3. Cloninger CR, Zohar AH. Personality and the perception of health and happiness. J Affect Disord. 2011;128:24-32.

4. Akiskal HS, Akiskal KK, Haykal RF, Manning JS, Connor PD. TEMPS-A: progress towards validation of a self-rated clinical version of the Temperament Evaluation of the Memphis, Pisa, Paris, and San Diego Autoquestionnaire. J Affect Disord. 2005;85:3-16.

5. Nakai $Y$, Inoue $T$, Toda $H$, et al. The influence of childhood abuse, adult stressful life events and temperaments on depressive symptoms in the nonclinical general adult population. J Affect Disord. 2014;158:101-107.

6. Kraepelin E. Psychiatrie, 8. Aufl. Leipzig: Barth; 1913.

7. World Health Organization. Assessment of Subjective Well-Being: The Subjective Well-Being lnventory. New Delhi, India: WHO Regional Office for South-East Asia; 1992.

8. Sarason IG, Johnson JH, Siegel JM. Assessing the impact of life changes: development of the Life Experiences Survey. J Consult Clin Psychol. 1978;46:932-946.

9. Matsumoto S, Akiyama T, Tsuda H, et al. Reliability and validity of TEMPS-A in a Japanese non-clinical population: application to unipolar and bipolar depressives. $J$ Affect Disord. 2005;85:85-92.
10. Sanders B, Becker-Lausen E. The measurement of psychological maltreatment: early data on the Child Abuse and Trauma Scale. Child Abuse Negl. 1995;19:315-323.

11. Tanabe H, Ozawa S, Goto K. Psychometric properties of the Japanese version of the Child Abuse and Trauma Scale (CATS). Paper presented at: The 9th Annual Meeting of the Japanese Society for Traumatic Stress Studies; Kobe; March 6; 2010. Japanese.

12. Tonan K, Sonoda A, Ono Y. Production of the subjective well-being inventory Japanese edition: its reliability and validity. Jpn J Health Psychol. 1995;8:12-19. Japanese.

13. Muramatsu K, Miyaoka H, Kamijima K, et al. The patient health questionnaire, Japanese version: validity according to the mini-international neuropsychiatric interview-plus. Psychol Rep. 2007;101:952-960.

14. Schermelleh-Engel K, Moosbrugger H, Müller H. Evaluating the fit of structural equation models: tests of significance and descriptive goodness-of-fit measures. MPR Online. 2003;8:23-74.

15. Alloy LB, Abramson LY, Smith JM, Gibb BE, Neeren AM. Role of parenting and maltreatment histories in unipolar and bipolar mood disorders: mediation by cognitive vulnerability to depression. Clin Child Fam Psychol Rev. 2006;9:23-64.

16. Binder EB, Bradley RG, Liu W, et al. Association of FKBP5 polymorphisms and childhood abuse with risk of posttraumatic stress disorder symptoms in adults. JAMA. 2008;299:1291-1305.

17. Heim C, NemeroffCB. The role of childhood trauma in the neurobiology of mood and anxiety disorders: preclinical and clinical studies. Biol Psychiatry. 2001;49:1023-1039.

18. Nakai Y, Inoue T, Chen C, et al. The moderator effects of affective temperaments, childhood abuse and adult stressful life events on depressive symptoms in the nonclinical general adult population. $J$ Affect Disord. 2015;187:203-210.

19. Toda $H$, Inoue $T$, Tsunoda $T$, et al. The structural equation analysis of childhood abuse, adult stressful life events, and temperaments in major depressive disorders and their influence on refractoriness. Neuropsychiatr Dis Treat. 2015;11:2079-2090.

20. McKee-Ryan F, Song Z, Wanberg CR, Kinicki AJ. Psychological and physical well-being during unemployment: a meta-analytic study. J Appl Psychol. 2005;90:53-76.

21. Hansson A, Hillerås P, Forsell Y. Well-being in an adult Swedish population. Soc Indic Res. 2005;74:313-325.

22. Wardenaar KJ, van Veen T, Giltay EJ, Zitman FG, Penninx BW. The use of symptom dimensions to investigate the longitudinal effects of life events on depressive and anxiety symptomatology. J Affect Disord. 2014;156:126-133.

23. Jeronimus BF, Ormel J, Aleman A, Penninx BW, Riese H. Negative and positive life events are associated with small but lasting change in neuroticism. Psychol Med. 2013;43:2403-2415.

24. Akiskal HS, Mendlowicz MV, Jean-Louis G, et al. TEMPS-A: validation of a short version of a self-rated instrument designed to measure variations in temperament. $J$ Affect Disord. 2005;85:45-52.

25. Rovai L, Maremmani AG, Rugani F, et al. Do Akiskal \& Mallya's affective temperaments belong to the domain of pathology or to that of normality? Eur Rev Med Pharmacol Sci. 2013;17:2065-2079.
Neuropsychiatric Disease and Treatment

\section{Publish your work in this journal}

Neuropsychiatric Disease and Treatment is an international, peerreviewed journal of clinical therapeutics and pharmacology focusing on concise rapid reporting of clinical or pre-clinical studies on a range of neuropsychiatric and neurological disorders. This journal is indexed on PubMed Central, the 'PsycINFO' database and CAS,
Dovepress

and is the official journal of The International Neuropsychiatric Association (INA). The manuscript management system is completely online and includes a very quick and fair peer-review system, which is all easy to use. Visit http://www.dovepress.com/testimonials.php to read real quotes from published authors. 